\title{
Trimethoprim in the Treatment of Urinary Infections in Hospital
}

\author{
R. N. GRÜNEBERG,* M.B., B.S., M.C.PATH. ; R. KOLBE, $†$ M.B., CH.B.
}

Brit. med. F., 1969, 1, 545-547

\begin{abstract}
Cummary : Success in the cure of urinary infections of hospital patients was compared for five-day courses of sulphamethoxazole alone, sulphamethoxazole plus onetenth its weight of trimethoprim, and sulphamethoxazole plus one-fifth its weight of trimethoprim (Septrin). The cure rates were $65 \%, 84 \%$, and $92 \%$ respectively. Fifty-four per cent. of 111 patients had urinary tract abnormalities. Forty-three per cent. of the causative organisms were sulphonamide-resistant in vitro. There were no major side-effects, though two patients had pruritus or a rash.

The degree of potentiation of sulphamethoxazole activity by one-fifth the weight of trimethoprim was so great that its cure rate of infections due to sulphonamideresistant organisms exceeded that of sulphamethoxazole alone used in infections due to sulphonamide-sensitive organisms. The degree of synergism between trimethoprim and sulphamethoxazole demonstrated in vitro against urinary organisms was directly related to the cure rate of the combination.
\end{abstract}

\section{Introduction}

Trimethoprim (2,4-diamino-5-(3', 4', 5'-trimethoxybenzyl) pyrimidine) is a member of a series of compounds first fully described by Roth, Falco, Hitchings, and Bushby (1962). It has marked antibacterial activity from its intense inhibition of the enzyme dihydrofolate reductase in bacteria, and therapeutic potential from its negligible binding of the similar enzyme in man. The enzyme is responsible for the conversion of dihydrofolate to tetrahydrofolate, a stage in a sequence leading to the synthesis of purines and deoxyribonucleic acid. This stage is immediately preceded in bacteria, but not in man, by the incorporation of para-aminobenzoic acid into dihydrofolic acid. This is blocked by sulphonamides, which might explain the observation that the antibacterial activities of trimethoprim and a sulphonamide are potentiated by each other (Bushby and Hitchings, 1968). The combination of trimethoprim and a sulphonamide is bactericidal, whereas sulphonamides alone are bacteriostatic.

There have been few clinical studies of trimethoprim. The only cases of urinary infection treated with this substance so far reported are of proteus infections (Schneider, Schwarzenberg, Cattan, Schlumberger, Amiel, and Mathé, 1965) and of klebsiella infections (Darrell, Garrod, and Waterworth, 1968).

In view of the interesting properties of trimethoprimsulphonamide combinations in vitro, we have performed a clinical trial comparing the effectiveness of combined therapy with that of a sulphonamide alone in the treatment of hospital patients with urinary infections.

\section{Methods}

The sole criterion of urinary infection was the presence of a pure growth of more than 100,000 organisms per ml. of urine as demonstrated by a surface-viable counting technique. Urine

\footnotetext{
* Senior Registrar in Bacteriology, Whittington Hospital, London N.19, and University College Hospital, London W.C.1. † Registrar in Clinical Pathology, Whittington Hospital, London N.19.
}

was collected in each case by a clean catch method, and promptly refrigerated until examination, which was always within two hours.

Sulphonamide-sensitivity tests were made on the urinary organisms by a disc method using a medium free of paraaminobenzoic acid. The inoculum density and the effectiveness of the discs were controlled in every test by using a known sulphonamide-sensitive organism. Minimum inhibitory concentrations of trimethoprim and sulphamethoxazole (Gantanol) for the urinary organisms were determined by means of a serial dilution method on solid media. A similar method was used in testing for the initial potentiation of activities of the two compounds in the relative concentrations of one part trimethoprim to 20 parts sulphamethoxazole. This ratio was accepted because trimethoprim is usually about 20 times more active than sulphamethoxazole in vitro. Bushby and Hitchings (1968) describe the methods in detail.

\section{The Trial}

After obtaining agreement of the respective clinicians in charge, 111 adult hospital inpatients with urinary tract infection were admitted to the trial. None was excluded because of anatomical abnormalities of the urinary tract or because of infections by organisms generally regarded as difficult to eradicate, such as Pseudomonas aeruginosa. Pregnant women were, however, excluded. The patients were allocated randomly to one of three groups. Every patient was treated for five days. Those in group 1 took sulphamethoxazole $1 \mathrm{~g}$. twice daily, those in group 2 took a combination of sulphamethoxazole $1 \mathrm{~g}$. and trimethoprim $100 \mathrm{mg}$. twice daily, and those in group 3 took a combination of sulphamethoxazole $800 \mathrm{mg}$. and trimethoprim $160 \mathrm{mg}$. twice daily. In other words the efficacy was compared of $10 \mathrm{~g}$. of sulphamethoxazole, $10 \mathrm{~g}$. of sulphamethoxazole plus $1 \mathrm{~g}$. (a 10 to 1 ratio) of trimethoprim, and $8 \mathrm{~g}$. of sulphamethoxazole plus $1.6 \mathrm{~g}$. (a 5 to 1 ratio) of trimethoprim. The criterion for cure was absence of the infecting organism from the urine on the eighth and fifteenth days ; that is to say, on the third and tenth day after treatment.

\section{Results}

Table I compares the patients allocated to the three treatment groups. The differences are unimportant. Table II compares the organisms infecting the patients in each group who completed the follow-up. Again the differences are unimportant. Sulphamethoxazole resistance in vitro was present in $43 \%$ of

TABLE I.-Comparison of Patients in the Three Treatment Groups

\begin{tabular}{|c|c|c|c|}
\hline & Group 1 & Group 2 & Group 3 \\
\hline $\begin{array}{l}\text { No. of patients completing study* } \ldots \\
\text { No. of female patients } . \\
\text { Average age (years) } \\
\text { No. of patients with “ urinary infec- } \\
\text { tion" symptoms } \ldots \\
\text { Previous history of urinary infection } \\
\text { (where elicitable) } . \\
\text { Recognizable predisposing cause to } \\
\text { infection (catheterization, urinary } \\
\text { tract abnormality, diabetes, etc.) }\end{array}$ & $\begin{array}{c}29 \\
21(72 \%) \\
62 \cdot 1 \\
19(66 \%) \\
68 \%\end{array}$ & $\begin{array}{c}37 \\
28(76 \%) \\
67 \cdot 4\end{array}$ & $\begin{array}{c}39 \\
30\left(\begin{array}{c}77 \% \\
65.8\end{array}\right. \\
16(41 \%) \\
59 \%\end{array}$ \\
\hline
\end{tabular}

* Five patients died and one other patient was not followed up. 
the organisms. In particular, sulphamethoxazole resistance among the organisms isolated from patients treated with sulphamethoxazole alone was present in $41 \%$.

TABLE II.-Comparison of Infecting Urinary Organisms in Each Treatment Group

\begin{tabular}{|c|c|c|c|}
\hline \multirow{2}{*}{ Organisms } & \multicolumn{3}{|c|}{ No. of Patients } \\
\hline & Group 1 & Group 2 & Group 3 \\
\hline 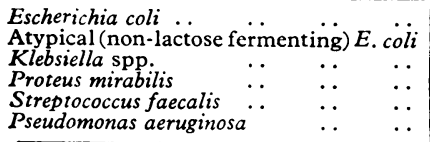 & $\begin{array}{r}18 \\
2 \\
6 \\
1 \\
1 \\
1\end{array}$ & $\begin{array}{r}19 \\
2 \\
8 \\
3 \\
4 \\
1\end{array}$ & $\begin{array}{r}25 \\
2 \\
5 \\
4 \\
2 \\
1\end{array}$ \\
\hline Total .. & 29 & 37 & 39 \\
\hline
\end{tabular}

Table III shows the results of treatment in each group. Group 1 received sulphamethoxazole alone and 5 of the 12 infected with sulphonamide-resistant organisms were nevertheless cured $(42 \%)$. Fourteen of the subjects with sulphonamidesensitive organisms were cured $(82 \%)$. The overall cure rate in this group was $65 \%$.

\begin{tabular}{|c|c|c|c|c|c|c|}
\hline & & & & \multicolumn{3}{|c|}{ No. of Patients } \\
\hline & & & & Group 1 & Group 2 & Group 3 \\
\hline $\begin{array}{l}\text { Cured .. } \\
\text { Failed treatment } \\
\text { Died to follow-up } \\
\text { Lost to follo }\end{array}$ & $\begin{array}{l}\cdots \\
\cdots \\
\cdots\end{array}$ & $\begin{array}{l}\cdots \\
\cdots \\
\cdots\end{array}$ & \begin{tabular}{l|l}
$\cdots$ & \\
$\cdots$ & \\
$\cdots$ &
\end{tabular} & $\begin{array}{c}19(65 \%) \\
10 \\
1 \\
-\end{array}$ & $\begin{array}{c}31(84 \%) \\
6 \\
2 \\
1 \\
\end{array}$ & $\begin{array}{c}36(92 \%) \\
3 \\
2 \\
-\end{array}$ \\
\hline \multicolumn{2}{|c|}{ Total completing treatment } & $\ldots$ & $\ldots$ & 29 & 37 & 39 \\
\hline
\end{tabular}

Fisher's exact probability test was used for all cases; the demonstrated superiority of the $1: 10$ ratio of trimethoprim to sulphamethoxazole (group 2) over sulphamethoxazole alone (group 1) did not quite reach conventional levels of significance $(\mathbf{P}=0.077)$. The superiority of the $1: 5$ ratio of trimethoprim and sulphamethoxazole (group 3) over sulphamethoxazole was convincing $(P=0.0067)$. A significant difference in cure rates produced by the two mixtures could not be detected $(P=0 \cdot 21)$. Table IV relates the cure rates of patients to the degree of antibacterial potentiation exhibited by the drug combination against their infecting organisms. The potentiation was measured in vitro and expressed as the ratio by which the minimum inhibitory concentration (M.I.C.) of sulphamethoxazole acting alone exceeded the M.I.C. of sulphamethoxazole plus onetwentieth part by weight of trimethoprim. If the ratio of the M.I.C. of sulphamethoxazole alone to sulphamethoxazole plus trimethoprim was $8: 1$, the potentiation is described as eightfold. The cure rate appears to be directly related to the degree of potentiation.

TABLE IV.-Results of Treatment with Sulphamethoxazole-trimethoprim in 68 Patients According to Degree of Potentiation of Sulphamethoxazole by Trimethoprim Demonstrated in vitro Against the Urinary Organism

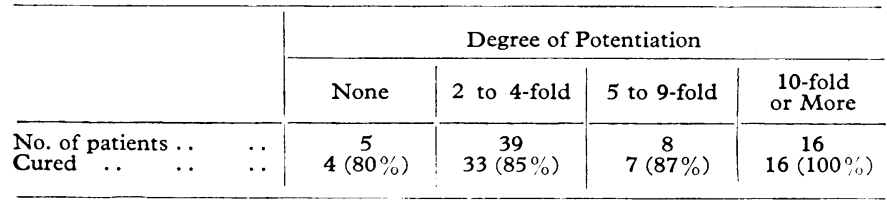

In the course of the trial 5 of the 111 patients died-two from carcinomatosis, one from a cerebrovascular accident, one from bronchopneumonia, and one from chronic renal failure.

\section{Discussion}

The trial showed that a five-day course of $0.32 \mathrm{~g}$. of trimethoprim plus $1.6 \mathrm{~g}$. of sulphamethoxazole daily was significantly superior to a five-day course of $2 \mathrm{~g}$. of sulphameth- oxazole alone daily. A similar comparison of $2 \mathrm{~g}$. of sulphamethoxazole daily and sulphamethoxazole plus a smaller proportion of trimethoprim showed the latter also to be superior, but the difference did not quite reach a significant level. The tendency for additional improvement to be related to increasing amounts of trimethoprim was suggested but not proved. The difficulty in proving this is magnified as the cure rate rises, and the number of patients required would far exceed those available for the study.

There is no certainty that some of the patients with cured infection would not have relapsed if the follow-up period had been for longer than 10 days. Nevertheless, the action of trimethoprim and sulphamethoxazole in sterilizing the urine of such a high proportion of hospital patients with infection is striking. The frequency of catheterization, urinary tract abnormalities, and diabetes exceeded $50 \%$ in all three treatment groups. In these unpromising circumstances a high cure rate is not to be expected with any present-day therapy, yet the overall cure rate achieved by the mixture of sulphamethoxazole with trimethoprim was $88 \%$. This result is similar to that obtained by treatment with either a sulphonamide or ampicillin of the much more responsive primary urinary tract infections in domiciliary practice (Grüneberg and Brumfitt, 1967).

Because of the high incidence of sulphonamide resistance in the whole series, expectation of a cure with the sulphonamide alone could not be high, though a degree of unpredictability in the sensitivity test is acknowledged in the $42 \%$ cure rate seen when using sulphamethoxazole alone against organisms with a reported in vitro resistance. Spontaneous resolution or the very high urinary concentration of the sulphonamide might explain this. The cure rate of sulphamethoxazole alone (82\%) was satisfactory when the sensitivity indicated that a cure could be expected. Nevertheless the addition of trimethoprim always improved on this cure rate without regard for the sulphonamide sensitivity of the organisms in the group as a whole. However, the cure rates of the drug combination were higher when the organisms were sulphonamide-sensitive (93\%) than when they were sulphonamide-resistant $(81 \%)$. These facts should be considered together with the finding that the degree of antibacterial potentiation in vitro runs closely parallel with the success of treatment.

The results of in vitro studies of the potentiation of sulphamethoxazole by trimethoprim may be expressed according to the extent by which the minimum inhibitory concentration (M.I.C.) of both drugs when acting together in the ratio of 20 parts sulphamethoxazole to one part trimethoprim is less than the M.I.C. of sulphamethoxazole alone for the individual organism. Table IV shows the results of such studies with the urinary organisms from 68 of the 76 patients who completed the period of follow-up after treatment with sulphamethoxazoletrimethoprim combinations. Only 5 of the 68 organisms $(7 \%)$ showed no enhancement of sulphamethoxazole activity when this was supplemented with trimethoprim, while 39 showed twofold to fourfold potentiation. Eight showed fivefold to ninefold potentiation, and 16 showed tenfold or more potentiation. Table IV also shows that the degree of potentiation demonstrated in vitro runs closely parallel with the outcome of treatment. The cure rate rises from $80 \%$ in patients whose infecting organisms show no evidence of greater response in the presence of both drugs than in the presence of sulphamethoxazole alone, through intermediate steps to a $100 \%$ cure rate in those patients whose organisms are tenfold or more more sensitive to the combination than to sulphamethoxazole alone.

Of the three patients with infections caused by Ps. aeruginosa, one was treated with sulphamethoxazole alone and failed to respond and two were treated with sulphamethoxazole and trimethoprim, and one was cured and the other was not. All three organisms were sulphonamide-sensitive in vitro.

Seven patients had infections caused by Str. faecalis. One was treated with sulphamethoxazole alone and failed to respond, 
while six were treated with sulphamethoxazole and trimethoprim and all six were cured. This observation is of interest because Str. faecalis is resistant to sulphamethoxazole but sensitive to trimethoprim.

Side-effects observed in the present series were few and trivial. One patient on sulphamethoxazole-trimethoprim $(10: 1)$ developed a pruritic rash on the limbs and trunk, which rapidly improved, and another patient on the same formulation developed pruritus without a rash. This patient was receiving a number of other drugs at the time, and the role of the sulphamethoxazole-trimethoprim in the production of the pruritus was very doubtful. The four patients out of 81 receiving trimethoprim-sulphamethoxazole who died during the course of this trial all died of causes unrelated to their treatment-two died of carcinomatosis, one with a cerebrovascular accident, and one with chronic renal failure. One patient on sulphamethoxazole alone died of bronchopneumonia.

The better formulation of sulphamethoxazole-trimethoprim appeared to be that containing the two drugs in the ratio $5: 1$, since this gave a cure rate of $92 \%$ compared with $84 \%$ for the $10: 1$ formulation. Because of the very low. failure rates with the two formulations of sulphamethoxazole-trimethoprim, formal tests of statistical significance for this difference are not applicable. The patients took two tablets twice a day for five days, and this appeared to be acceptable. The smaller amount of sulphamethoxazole in the $1: 5$ combination (Septrin) tablets than in the other two treatments was to ensure a suitable size of tablet so that the pattern of tablet taking in the three groups was acceptable to all patients.
In view of the very high cure rate in urinary infections in hospital inpatients combined with a low incidence of side-effects of a non-serious nature and an acceptable formulation, we consider that sulphamethoxazole-trimethoprim should be more widely used. There appears to be no reason why it should not also be used in domiciliary practice. We have no experience of the use of this combination in the treatment of urinary infections in children or in pregnant women and do not advocate its use in these infections until more is known of its sideeffects.

We are grateful to our clinical colleagues at the Whittington Hospital for permission to treat patients under their care and to the nursing staff and the technicians in the bacteriology laboratory for their enthusiastic help. Dr. A, F. Mohun, Dr. J. G. A. McSorley, and Dr. H. E. S. Pearson gave helpful advice and encouragement. Dr. A. S. E. Fowle, of the Wellcome Research Foundation, supplied the sulphonamide and trimethoprim used in the trial and provided valuable information and assistance throughout. Dr. S. R. M. Bushby of the Wellcome Foundation kindly performed the in-vitro studies on potentiation of sulphonamide by trimethoprim.

\section{REFERENCES}

Bushby, S. R. M., and Hitchings, G. H. (1968). Brit. F. Pharm., 33, 72. Darrell, J. H., Garrod, L. P., and Waterworth, P. M. (1968). F. clin. Path., 21, 202.

Grüneberg, R. N., and Brumfitt, W. (1967). Brit. med. F., 3, 649.

Roth, B., Falco, E. A., Hirchings, G. H., and Bushby, S. R. M. (1962). 7. med. pharm. Chem., 5, 1103.

Schneider, M., Schwarzenberg, L., Cattan, A., Schlumberger, J.-R., Amiel, J.-L., and Mathé, G. (1965). Presse méd., 73, 893.

\title{
Relationship of a Degenerative Tropical Neuropathy to Diet Report of a Field Survey
}

\author{
B. O. OSUNTOKUN,* M.B., B.S., M.R.C.P. ; G. L. MONEKOSSO, † M.D., F.R.C.P.ED., D.T.M.\&H. \\ J. WILSON $\ddagger$ M.B., PH.D., M.R.C.P.
}

Brit. med. F., 1969, 1, 547-550

\begin{abstract}
Summary : A survey of neurological abnormalities in $N$ two Nigerian villages preselected for their differing consumption of cassava has shown that a degenerative neuropathy occurs with relatively high frequency in the village (Ososa) where cassava consumption was high.

It is suggested that eating cassava results in exposure to cyanide, as shown by a raised plasma thiocyanate level, and that the latter, together with other factors, may contribute to the pathogenesis of "tropical neuropathy."
\end{abstract}

\section{Introduction}

It has been suggested that chronic cyanide intoxication from dietary sources may be a contributory factor in the aetiology of a degenerative neurological disorder in Nigeria (Clark, 1935; Monekosso and Annan, 1964 ; Monekosso and Wilson, 1966 ; Osuntokun, 1968). The main features of the fully developed

* Physician in Neurology, University College Hospital, Ibadan, Nigeria. † Professor of Medicine, University of Lagos, Nigeria. Present address: W.H.O. Professor of Medicine and Dean of the Faculty of Medicine, University of East Africa, Dar-es-Salaam, Tanzania.

¥ Member of Scientific Staff, M.R.C. Clinical Genetics Research Unit, Institute of Child Health and Institute of Neurology, London W.C.1. "ataxic syndrome"-optic atrophy, nerve deafness, and sensory spinal ataxia - have been well documented (Money, 1958). Tropical amblyopia, probably a fragmentary form of the fully developed syndrome, was first studied in Nigeria by Moore (1930, 1932, 1934a, 1934b, 1937), who associated it with poor diet and thought that a nutritional deficiency of riboflavine and/or a dietary toxin should be implicated.

The association of angular stomatitis, glossitis, and scrotal dermatitis with tropical amblyopia has been confirmed by observers in different countries in peacetime and during the second world war (Denny-Brown, 1947 ; Smith and Woodruff, 1950 ; Cruickshank, 1952 ; Monekosso, 1963 ; Monekosso and Ashby, 1963) ; these mucocutaneous deficiency lesions have also been recognized in association with the fully developed ataxic syndrome (Scott, 1918 ; Landor and Pallister, 1935; DennyBrown, 1947 ; Knüttgen, 1955 ; Money, 1958 ; Ebrahim and Haddock, 1964 ; Monekosso and Annan, 1964). Many observers have therefore sought a link between vitamin-B-complex deficiency (especially of riboflavine) and the ataxic neurological syndrome. Although in a number of respects the condition resembles clinically subacute combined degeneration as seen in European communities, serum vitamin $B_{12}$ levels are not reduced. Moreover, clinical "ariboflavinosis" is very common 